\title{
THE ROLLING BALL PROBLEM ON THE PLANE REVISITED
}

\author{
LAURA M. O. BISCOLLA, JAUME LLIBRE AND WALDYR M. OLIVA
}

\begin{abstract}
By a sequence of rollings without slipping or twisting along segments of an straight line of the plane a spherical ball of unit radius has to be transferred from an initial state to an arbitrary final state taking into account the orientation of the ball. We provide a new proof that with at most 3 moves we can go from a given initial state to an arbitrary final state. The first proof of this result is due to Hammersley [3]. His proof is more algebraic than ours which is more geometric.
\end{abstract}

\section{IntroduCtion AND STATEMENT OF THE RESUlts}

The rollings of a spherical ball $B$ of unit radius over the plane $\mathbb{R}^{2}$ suggest the consideration of some special kinematic (virtual) motions. A state of the ball is defined as to be the pair formed by the point of contact between $B$ and $\mathbb{R}^{2}$ and by a positive orthonormal frame attached to $B$. So the set of all the states is identified with the manifold $\mathbb{R}^{2} \times S O(3)$, where $S O(3)$ denotes the group of all orthogonal $3 \times 3$ matrices with positive determinant.

A move is a smooth path on $\mathbb{R}^{2} \times S O(3)$ corresponding to a rolling of $B$ on $\mathbb{R}^{2}$ without slipping or twisting along a straight line of the plane $\mathbb{R}^{2}$. No slipping in the rolling means that at each instant the point of contact between $B$ and $\mathbb{R}^{2}$ has zero velocity; no twisting means that, at each instant, the axis of rotation must be parallel to the plane $\mathbb{R}^{2}$.

According with John M. Hammersley the following problem was proposed by David Kendall in the 1950s: What is the number $N$ of moves necessary and sufficient to reach any final state of $\mathbb{R}^{2} \times S O(3)$ starting at a given initial state? In an interesting paper written in 1983 Hammersley [3] shows that $N=3$ after using, strongly, the theory of quaternions.

The following historical considerations we quote from [3] p.112: The original version of the question set (by David Kendall in the 1950s) for 18-year-old schoolboys, invited candidates to investigate how two moves, each of length $\pi$, would change the ball's orientation; and to deduce in the first place that $N \leq 11$, and in the second place that $N \leq 7$. Candidates scored bonus marks for any improvement on 7 moves. When he first set the question, Kendall knew that $N \leq 5$; but, interest being aroused amongst professional mathematicians at Oxford, he and others soon discovered that the answer must be either $N=3$ or $N=4$. But in the 1950 s nobody could decide between these two possibilities. There was renewed interested in the 1970s, and not only amongst professional mathematicians: for example the President of Trinity (a distinguished biochemist) spent some time rolling a ball around his drawing room floor in search of empirical insight. In 1978, while delivering the opening address to the first Australasian Mathematical Convention, I posed the problem to mathematicians down under; but I have not subsequently received a solution from them. So this is an opportunity to publish the solution.

In the present paper we shall prove that $N=3$ but the proof provided here is more geometric than the original one which is more algebraic and uses strongly the theory of quaternions.

A state $(P, M) \in \mathbb{R}^{2} \times S O(3)$ of the spherical ball on the plane $\mathbb{R}^{2}$ means that the contact point of the ball with the $(x, y)$-plane is the point $P$ of $\mathbb{R}^{2}$ and its orientation is given by

1991 Mathematics Subject Classification. Primary 58E25, 93B27.

Key words and phrases. Control theory, rolling ball. 
the orthonormal frame $(I, J, K)$ of $\mathbb{R}^{3}$ where the vectors $I, J$ and $K$ are given by the first, second and third column of the matrix $M$, respectively. Moreover the state $(P, M)$ is also denoted by $(P,(I, J, K))$. We denote the orthonormal frame $(I, J, K)$ associated to the $3 \times 3$ identity matrix, $I d$, by $(i, j, k)$.

The paper is organized as follows. In section 2 we recall the Euler angles, and using these angles we can get an easy representation of the elements of $S O(3)$. In section 3 we prove that $N=3$. More precisely, Theorem 3 proves that with 3 moves one goes from the initial state $\left(P_{0},(i, j, k)\right)$ to a final state $\left(P_{0},(I, J, k)\right)$. To simplify notation the previous phrase is simplified as

$$
\left(P_{0},(i, j, k)\right) \rightarrow\left(P_{0},(I, J, k)\right) \text { in Theorem } 3 .
$$

Analogously we prove:

$$
\begin{aligned}
& \left(P_{0},(i, j, k)\right) \rightarrow\left(P_{0},(I, J,-k)\right) \text { in Proposition } 5 \\
& \left(P_{0},(i, j, k)\right) \rightarrow\left(P_{0},(I, J, K)\right) \text { in Theorem } 7 .
\end{aligned}
$$

Now, if $P_{1} \neq P_{0}$ we also prove:

$$
\begin{aligned}
& \left(P_{0},(i, j, k)\right) \rightarrow\left(P_{1},(I, J, k)\right) \text { in Proposition } 4, \\
& \left(P_{0},(i, j, k)\right) \rightarrow\left(P_{1},(I, J,-k)\right) \text { in Proposition } 6, \\
& \left(P_{0},(i, j, k)\right) \rightarrow\left(P_{1},(I, J, K)\right) \text { in Theorem } 8 .
\end{aligned}
$$

We observe that Theorem 3 means that we obtain the so called elimination of the spin discrepancy.

The two main reasons for revisiting the proof of the result mentioned as "Kendall problem" are: first, the paper written by Hammersley is hard to find in the mathematical literature, and second, the proof that appear in [3] of the section "7. Even version : case 1 ", which corresponds to our Theorem 7, presents, as far as we could understand, in pages 121 and 122, some arguments which are not completely clear.

\section{EULER ANGLES}

According to Euler's rotation theorem, any rotation of $\mathbb{R}^{3}$, i.e. any element of the $S O(3)$, may be described using three angles. If the rotations are written in terms of rotation matrices $R_{3}(a), R_{1}(b)$ and $R_{3}(c)$, then a general rotation $M \in S O(3)$ can be written as

$$
M=R_{3}(c) R_{1}(b) R_{3}(a),
$$

where

$$
R_{3}(a)=\left(\begin{array}{ccc}
\cos a & \sin a & 0 \\
-\sin a & \cos a & 0 \\
0 & 0 & 1
\end{array}\right), \quad R_{1}(b)=\left(\begin{array}{ccc}
1 & 0 & 0 \\
0 & \cos b & \sin b \\
0 & -\sin b & \cos b
\end{array}\right)
$$

The three angles giving the three rotation matrices are called Euler angles. There are several conventions for Euler angles, depending on the axes about which the rotations are carried out. The so-called $x$-convention is the one that we have used and is the most common definition. In this convention the rotation is given by Euler angles $(a, b, c)$ where the first rotation is by an angle $a$ about the $z$-axis, the second is by an angle $b$ in $[0, \pi]$ about the $x$-axis and the third is by an angle $c$ about the $z$-axis.

The Euler angles are related with the quaternions. The quaternions were used by Hammersley in his study of the ball problem. For more details about the Euler angles see for instance [2]. 


\section{Three MOVES ARE SUFFICIENT ON $\mathbb{R}^{2}$}

In this section we shall prove that $N=3$.

First we formulate explicitly a move of the spherical ball from the initial point $P_{0} \in \mathbb{R}^{2}$ with orientation $M_{0} \in S O(3)$ to the point $P_{1} \in \mathbb{R}^{2}$, always assuming $P_{1} \neq P_{0}$. Let $(x, y)$ be a given cartesian coordinate system of $\mathbb{R}^{2}$ with origin at $P_{0}$, i.e. $P_{0}=(0,0)$. Without loss of generality we can assume in all this section that $P_{0}=(0,0)$. As usual, we shall denote the polar coordinates of $P_{1}$ with origin at $P_{0}$ as $(r, \theta)$, where $\theta$ is measured in counterclockwise with respect to the positive $x$-axis. In what follows we simply say that $(r, \theta)$ are the polar coordinates of $P_{1}$ with respect to $P_{0}$. Now after the move through the segment starting at $P_{0}$ and ending at $P_{1}$ the orientation $M_{1} \in S O(3)$ at $P_{1}$ is given by

$$
M_{1}=R_{3}(-\theta) R_{2}(r) R_{3}(\theta) M_{0},
$$

where

For instance note that

$$
R_{2}(r)=\left(\begin{array}{ccc}
\cos r & 0 & \sin r \\
0 & 1 & 0 \\
-\sin r & 0 & \cos r
\end{array}\right)
$$

$$
R_{1}(a)=R_{3}(-\pi / 2) R_{2}(a) R_{3}(\pi / 2)
$$

We remark that the motion (1) is a rotation around the vector $e=(\cos (\pi / 2-\theta), \sin (\pi / 2-$ $\theta), 0$ ) of angle $r$, and we write it as

$$
\mathcal{R}(e, r) .
$$

It is well known that the rotation matrix $\mathcal{R}(e, \delta)$ of angle $\delta$ around an axis through the origin with direction $e=(u, v, w)$ is

$$
\frac{1}{L^{2}}\left(\begin{array}{ccc}
u^{2}+\left(v^{2}+w^{2}\right) \cos \delta & u v(1-\cos \delta)-w L \sin \delta & u w(1-\cos \delta)+v L \sin \delta \\
u v(1-\cos \delta)+w L \sin \delta & v^{2}+\left(u^{2}+w^{2}\right) \cos \delta & v w(1-\cos \delta)-u L \sin \delta \\
u w(1-\cos \delta)-v L \sin \delta & v w(1-\cos \delta)+u L \sin \delta & w^{2}+\left(u^{2}+v^{2}\right) \cos \delta
\end{array}\right),
$$

where $L=\sqrt{u^{2}+v^{2}+w^{2}}$.

The following two lemmas will be very useful. The first one allows to pass in 2 moves from the state $\left(P_{0},(I, J, K)\right)$ to an arbitrary state of the form $\left(P_{1},(I, J, K)\right)$ with $P_{1} \neq P_{0}$.

Lemma 1. Let $M \in S O(3)$ and $P_{0}, P_{1} \in \mathbb{R}^{2}$ with $P_{0} \neq P_{1}$. Then we can go from the initial state $\left(P_{0}, M\right)$ to the final state $\left(P_{1}, M\right)$ with 2 moves.

Proof. For doing the proof it is not restrictive to assume that $P_{0}$ is the origin of the cartesian coordinates and that $P_{1}$ is over the positive $x$-axis.

Let $(r, 0)$ be the polar coordinates of $P_{1}$ with respect to $P_{0}$. Take a positive integer $n$ such that $2 \pi n \geq r$ and let $\bar{P}$ be the point of polar coordinates $(\bar{r}, \bar{\theta})=(2 \pi n, \arccos (r /(4 \pi n)))$ with respect to $P_{0}$. It is easy to check that

(i) $d\left(\bar{P}, P_{0}\right)=d\left(\bar{P}, P_{1}\right)=\bar{r}$ where $d$ denotes the Euclidean distance of $\mathbb{R}^{2}$;

(ii) $(\bar{r},-\bar{\theta})$ are the polar coordinates of $P_{1}$ with respect to $\bar{P}$ (i.e. we can think that $\bar{P}$ is the new origin of coordinates and that $\bar{\theta}$ is measured in counterclockwise with respect to a parallel ray to the positive $x$-axis starting at $\bar{P})$; and

(iii) $\left(R_{3}(\bar{\theta}) R_{2}(\bar{r}) R_{3}(-\bar{\theta})\right)\left(R_{3}(-\bar{\theta}) R_{2}(\bar{r}) R_{3}(\bar{\theta})\right)=I d$.

Note that from (i) the points $P_{0}, \bar{P}$ and $P_{1}$ are the vertices of an isosceles triangle.

Now from the definition of move (see (1)), it follows that doing the two moves: first the move starting at $P_{0}$ and ending at $\bar{P}$, and second the move starting at $\bar{P}$ and ending at $P_{1}$, the state of the ball pass from $\left(P_{0}, M\right)$ to $\left(P_{1}, M\right)$.

As usual we denote by $\mathbb{S}^{1}=\mathbb{R} /(2 \pi \mathbb{Z})$ the circle. The following result allows to pass in 2 moves from an state $\left(P_{0},(i, j, k)\right)$ to a convenient state of the form $\left(P_{2},(I, J, k)\right)$. This result also appears in Biscolla [1]. 
Lemma 2. Given $a \in \mathbb{S}^{1}$ and a point $P_{0} \in \mathbb{R}^{2}$ we consider the point $P_{1}$ with polar coordinates $((2 n+1) \pi, b)$ with respect to $P_{0}$, and the point $P_{2}$ with polar coordinates $((2 m+1) \pi, b-a / 2)$ with respect to $P_{1}$, where $n$ and $m$ are non-negative integers. Then the following statements hold:

(a) The geometrical locus of all points $P_{2}$ with $b \in \mathbb{S}^{1}$ is a circle centered at $P_{0}$ of radius

$$
\pi \sqrt{2(1+2 n(1+n)+2 m(1+m)+(1+2 n)(1+2 m) \cos (a / 2)}) .
$$

(b) For any $b \in \mathbb{S}^{1}$ the initial state $\left(P_{0}, I d\right)$ pass to the final state $\left(P_{2}, R_{3}(a)\right)$ after doing 2 moves: first the move starting at $P_{0}$ and ending at $P_{1}$, and second the move starting at $P_{1}$ and ending at $P_{2}$.

Proof. Without loss of generality we assume that the point $P_{0}$ is at the origin of the cartesian coordinates. Then an easy computation shows that the cartesian coordinates of $P_{2}$ are

$$
\pi((1+2 n) \cos b+(1+2 m) \cos (b-a / 2),(1+2 n) \sin b+(1+2 m) \sin (b-a / 2)) .
$$

Hence statement (a) follows.

Since $\left(R_{3}(a / 2-b) R_{2}((2 m+1) \pi) R_{3}(b-a / 2)\right)\left(R_{3}(-b) R_{2}((2 n+1) \pi) R_{3}(b)\right)=R_{3}(a)$ for all $b \in \mathbb{S}^{1}$, we get statement (b).

The next result allows to go with 3 moves from the state $\left(P_{0},(i, j, k)\right)$ to the state $\left(P_{0},(I, J, k)\right)$

Theorem 3. Given $P_{0} \in \mathbb{R}^{2}$ and $a \in \mathbb{S}^{1}$ we can pass from the initial state $\left(P_{0}, I d\right)$ to the final state $\left(P_{0}, R_{3}(a)\right)$ doing 3 moves.

Proof. Without loss of generality we assume that $P_{0}$ is at the origin of the cartesian coordinates, and that $a \in(0,2 \pi)$.

Now we shall provide the proof that 3 moves are sufficient. We first do the move: starting at origin and ending at the point $P_{1}=(r,-\theta)$ in polar coordinates with respect to $P_{0}$, for a convenient $\theta \in(\pi / 2-a / 4, \pi / 2)$ and

$$
r=\arccos (\cot \theta \tan (\theta+a / 2)) .
$$

The second move starts at $P_{1}$ and ends at the point $P_{2}=(2 r \sin (\pi / 2-\theta), \pi)$ in polar coordinates with respect to $P_{1}$. Finally the third move starts at $P_{2}$ and ends at $P_{0}=(r, \theta)$ in polar coordinates with respect to $P_{2}$.

The angle $\theta \in(\pi / 2-a / 4, \pi / 2)$ is chosen satisfying the equation

$$
\begin{aligned}
f(\theta)= & \arccos (\csc \theta \sin (\theta+a / 2)- \\
& \cos \theta \arccos (\cot \theta \tan (\theta+a / 2))=0 .
\end{aligned}
$$

It is easy to check that $f(\pi / 2-a / 4)=-\pi \sin (a / 4)<0, f(\pi / 2)=a / 2>0$, and $f$ is well defined in the interval $[\pi / 2-a / 4, \pi / 2]$, so by continuity there exists at least one $\theta \in(\pi / 2-a / 4, \pi / 2)$ satisfying equation (4). In fact the derivative of $f(\theta)$ is positive in $[\pi / 2-a / 4, \pi / 2]$, so there is a unique $\theta \in(\pi / 2-a / 4, \pi / 2)$ satisfying equation (4), but this uniqueness is not necessary in the proof.

These 3 moves take place on the sides of an isosceles triangle, being $P_{0}$ the vertex between the two equal sides of the triangle, the length of the equal sides is $r$, and the angle between the two equal sides is equal to $\pi-2 \theta$.

If we do these 3 moves, according with (1), the orientation of the ball changes as follows

$$
\begin{aligned}
& \left(R_{3}(-\theta) R_{2}(r) R_{3}(\theta)\right)\left(R_{3}(\pi) R_{2}\left(2 r \sin \left(\frac{\pi}{2}-\theta\right)\right) R_{3}(\pi)\right) \\
& \left(R_{3}(\theta) R_{2}(r) R_{3}(-\theta)\right) I d .
\end{aligned}
$$


We must show that this matrix is equal to $R_{3}(a)$, and the proposition would be proved. To prove the equality between the previous two matrices is equivalent to show the matrix

$$
\begin{aligned}
A= & \left(R_{3}(\pi) R_{2}\left(2 r \sin \left(\frac{\pi}{2}-\theta\right)\right) R_{3}(\pi)\right)\left(R_{3}(\theta) R_{2}(r) R_{3}(-\theta)\right)- \\
& \left(R_{3}(-\theta) R_{2}(-r) R_{3}(\theta)\right) R_{3}(a)
\end{aligned}
$$

is identically zero.

We denote by $a_{i j}$ the element in the row $i$ and in the column $j$ of the $3 \times 3$ matrix $A$. Then

$$
a_{33}=\sin (r \cos \theta)(\cos \theta \cos (r \cos \theta) \sin r-\cos r \sin (r \cos \theta))=0 .
$$

It easy to check that $\sin (r \cos \theta) \neq 0$, otherwise we get easily a contradiction with the fact that $A \equiv 0$. Therefore

$$
\cos \theta \cos (r \cos \theta) \sin r-\cos r \sin (r \cos \theta)=0 .
$$

From this equation we have that

$$
\sin (r \cos \theta)=\cos \theta \cos (r \cos \theta) \tan r
$$

if $\cos r \neq 0$. The equation $a_{21}=0$ is

$$
-\cos r \sin \theta(\cos \theta+\cos (\theta+a))+\cos \theta(\sin \theta+\sin (\theta+a))=0 .
$$

From it we get

$$
\cos r=\frac{\cos \theta(\sin \theta+\sin (\theta+a))}{\sin \theta(\cos \theta+\cos (\theta+a))},
$$

if $\sin \theta(\cos \theta+\cos (\theta+a)) \neq 0$. Now substituting $\sin (r \cos \theta)$ and $\cos r$ in the matrix $A$ we get that $a_{i j}=0$ for all $i, j=1,2,3$.

In short in order that $A \equiv 0$ it is sufficient to choose $r$ and $\theta$ satisfying (5), (6), $\cos r \neq 0$ and $\sin \theta(\cos \theta+\cos (\theta+a)) \neq 0$. Computing $r$ from (6) we get (3). Now substituting $\sin (r \cos \theta)$ and $r$ into $a_{32}=0$ we obtain

$$
\csc ^{2} \theta g(\theta)=0
$$

where

$$
\begin{aligned}
g(\theta)= & \cos (2 \theta) \cos ^{2}(\arccos (\cot \theta \tan (\theta+a / 2)) \cos \theta)-\cos (2 \theta+a)+ \\
& \sin ^{2}(\arccos (\cot \theta \tan (\theta+a / 2) \cos \theta) \\
= & 2 \sin ^{2}(\theta+a / 2)-2 \cos ^{2}\left(\arccos (\cot \theta \tan (\theta+a / 2) \cos \theta) \sin ^{2} \theta\right.
\end{aligned}
$$

Since $\theta \in(\pi / 2-a / 4, \pi / 2)$ we have that $\csc \theta \neq 0$, so $g(\theta)=0$. But in order that $g(\theta)=0$ it is sufficient that

$$
\sin (\theta+a / 2)-\cos (\arccos (\cot \theta \tan (\theta+a / 2) \cos \theta) \sin \theta=0 .
$$

That is that $f(\theta)=0$.

Summarizing in order that $A \equiv 0$ it is sufficient to solve the two equations (3) and (6) with respect to $\theta \in(\pi / 2-a / 4, \pi / 2)$ and $r>0$, and that the solution $(r, \theta)$ satisfies $\cos r \neq 0$ and $\sin \theta(\cos \theta+\cos (\theta+a)) \neq 0$. From (6) $\cos r \neq 0$ is equivalent to $\cos \theta(\sin \theta+\sin (\theta+a)) \neq$ 0 . Since $\theta \in(\pi / 2-a / 4, \pi / 2)$ it is easy to check that $\sin \theta(\cos \theta+\cos (\theta+a)) \neq 0$ and $\cos \theta(\sin \theta+\sin (\theta+a)) \neq 0$. Hence the theorem is proved.

The next result allows to go with 3 moves from the state $\left(P_{0},(i, j, k)\right)$ to an arbitrary state of the form $\left(P_{1},(I, J, k)\right)$.

Proposition 4. Given $P_{0}, P_{1} \in \mathbb{R}^{2}$ with $P_{0} \neq P_{1}$ and $a \in \mathbb{S}^{1}$ we can pass from the initial state $\left(P_{0}, I d\right)$ to the final state $\left(P_{1}, R_{3}(a)\right)$ doing 3 moves. 
Proof. Without loss of generality we assume that $P_{0}$ is at the origin of the cartesian coordinates. Then by Lemma 2 there are infinitely many circles centered at $P_{0}$ whose radii increase tending to infinity. We can pass with 2 moves from the point $P_{0}$ to any point of these circles, and on these points the orientation of the spherical ball is $R_{3}(a)$.

Clearly we can pass with 1 move from the point $P_{1}$ to the points of the circles centered at $P_{1}$ with radii $2 l \pi$ with $l=1,2, \ldots$, and on these points the orientation of the spherical ball is the same than in $P_{1}$, i.e. $R_{3}(a)$.

Since the two families of circles the one centered at $P_{0}$ and the other centered at $P_{1}$ intersect, it follows that we can pass from the initial state $\left(P_{0}, I d\right)$ to the final state $\left(P_{1}, R_{3}(a)\right)$ doing 3 moves.

The next result allows to go with 3 moves from the state $\left(P_{0},(i, j, k)\right)$ to an arbitrary state of the form $\left(P_{0},(I, J,-k)\right)$. First we introduce some notation. It is clear that any orthonormal frame $(I, J,-k)$ can be given by a rotation matrix of the form

$$
R_{3}^{*}(a)=\left(\begin{array}{ccc}
-\cos a & -\sin a & 0 \\
-\sin a & \cos a & 0 \\
0 & 0 & -1
\end{array}\right)
$$

for a convenient $a \in \mathbb{S}^{1}$.

Proposition 5. Given $P_{0} \in \mathbb{R}^{2}$ and $a \in \mathbb{S}^{1}$ we can pass from the initial state $\left(P_{0}, I d\right)$ to the final state $\left(P_{0}, R_{3}^{*}(a)\right)$ doing 3 moves.

Proof. Without loss of generality we assume that $P_{0}$ is at the origin of the cartesian coordinates. We first do the move: starting at origin and ending at the point $P_{1}=(\pi, a / 2)$ in polar coordinates with respect to $P_{0}$. Then the orientation of the ball at $P_{1}$ is $R_{3}(-a / 2) R_{2}(\pi)$ $R_{3}(a / 2)=R_{3}^{*}(a)$.

Now we can pass from the initial state $\left(P_{1}, R_{3}^{*}(a)\right)$ to the final state $\left(P_{0}, R_{3}^{*}(a)\right)$ doing two moves by using Lemma 1 . Hence the proposition is proved.

In what follows we shall see that we can go with 3 moves from the state $\left(P_{0},(i, j, k)\right)$ to an arbitrary state of the form $\left(P_{1},(I, J,-k)\right)$.

Proposition 6. Given $P_{0}, P_{1} \in \mathbb{R}^{2}$ with $P_{0} \neq P_{1}$ and $a \in \mathbb{S}^{1}$ we can pass from the initial state $\left(P_{0}, I d\right)$ to the final state $\left(P_{1}, R_{3}^{*}(a)\right)$ doing 3 moves.

Proof. Without loss of generality we assume that $P_{0}$ is at the origin of the cartesian coordinates. We first do the move: starting at origin and ending at the point $\bar{P}=(\pi, a / 2)$ in polar coordinates with respect to $P_{0}$. Then the orientation of the ball at $\bar{P}$ is $R_{3}(-a / 2) R_{2}(\pi) R_{3}(a / 2)=$ $R_{3}^{*}(a)$.

Now we can pass from the initial state $\left(\bar{P}, R_{3}^{*}(a)\right)$ to the final state $\left(P_{1}, R_{3}^{*}(a)\right)$ doing two moves by using Lemma 1 . Hence the proposition is proved.

We will show that we can go with 3 moves from the state $\left(P_{0},(i, j, k)\right)$ to the state $\left(P_{0},(I, J, K)\right)$, by assuming that $k$ and $K$ are linearly independent.

Theorem 7. Given $P_{0} \in \mathbb{R}^{2}$ and $M \in S O(3)$ with the third column of $M$ different from $\pm k$, we can pass from the initial state $\left(P_{0}, I d\right)$ to the final state $\left(P_{0}, M\right)$ doing 3 moves.

Proof. As usual we can suppose that $P_{0}$ is the origin of the cartesian coordinates. Let $(I, J, K)$ be the orthonormal frame associated to the rotation matrix $M=R_{3}(c) R_{1}(b) R_{3}(a)$, with $a, c \in \mathbb{S}^{1}$ and $b \in[0, \pi]$. We recall that from the definition of the Euler angles, that $b$ is the angle between the vectors $k$ and $K$. From the hypotheses $k$ and $K$ are linearly independent. Moreover we can think $M$ as a rotation around an eigenvector $v$ with eigenvalue 1 , and we know that $M$ moves $k$ into $K, j$ into $J$ and $i$ into $I$. 
Assume that the vector $v$ is horizontal, i.e. $v$ is in the plane generated by the vectors $\{i, j\}$. Now after rolling the unitary ball around the vector $v$ in the direction $v^{T}$ (the orthogonal vector to $v)$ a distance $b$, we see that the ball goes from the initial state $\left(P_{0}, I d\right)$ to a state $\left(P_{1}, M\right)$ because $k$ goes to $K$ and, because the ortogonal plane to $v$ is invariant under $M$, simultaneously $i$ goes to $I$ and $j$ goes to $J$. Now applying Lemma 1 to the ball at $\left(P_{1}, M\right)$ we get the state $\left(P_{0}, M\right)$ with two more moves. Then we go from $\left(P_{0}, I d\right)$ to $\left(P_{0}, M\right)$ with 3 moves.

In the rest of the proof we suppose that $v=\left(v_{1}, v_{2}, v_{3}\right)$ is not horizontal. So $v_{3} \neq 0$, and we can take $v_{3}=1$. Let $\pi$ be the plane through $P_{0}$ (the origin) orthogonal to the vector $v$. The equation of this plane $\pi$ is $v_{1} x+v_{2} y+z=0$. Since $k$ and $K$ are linearly independent, we have $v_{1}^{2}+v_{2}^{2} \neq 0$, otherwise $v=(0,0,1)$ and $M$ cannot send $k$ to $K$ because they are linearly independent. We choose the coordinates $(x, y, z)$ so that the intersection line between the plane $\pi$ and the horizontal plane be the $x$-axis. Therefore the plane $\pi$ has equation $v_{2} y+z=0$ with $v_{2} \neq 0$. Hence in the new coordinates $v=\left(0, v_{2}, 1\right)$, and let $\phi$ be the angle of the rotation $M$ around the vector $v$. Denote by $\varphi$ the norm of $v$, i.e. $\varphi^{2}=1+v_{2}^{2}$.

Choose on the plane $\pi$ the two unitary vectors

$$
\begin{aligned}
& u_{1}(0)=\left(\cos \frac{\phi}{4},-\frac{1}{\varphi} \sin \frac{\phi}{4}, \frac{v_{2}}{\varphi} \sin \frac{\phi}{4}\right), \\
& u_{2}(0)=\left(\cos \frac{\phi}{4}, \frac{1}{\varphi} \sin \frac{\phi}{4},-\frac{v_{2}}{\varphi} \sin \frac{\phi}{4}\right) .
\end{aligned}
$$

Note that the vectors $u_{1}(0)$ and $u_{2}(0)$ from an angle $\phi / 4$ with the $x$-axis.

By rotating the vectors $u_{1}(0)$ and $u_{2}(0)$ an angle $\lambda$ on the plane $\pi$ we get the unitary vectors

$$
\begin{aligned}
& u_{1}(\lambda)=\left(\cos l, \frac{1}{\varphi} \sin l,-\frac{v_{2}}{\varphi} \sin l\right), \\
& u_{2}(\lambda)=\left(\cos L, \frac{1}{\varphi} \sin L,-\frac{v_{2}}{\varphi} \sin L\right),
\end{aligned}
$$

where $l=\lambda-\phi / 4$ and $L=\lambda+\phi / 4$.

We introduce now the horizontal vectors

$$
\begin{aligned}
& h_{1}(\lambda)=k \wedge u_{1}(\lambda)=\left(-\frac{1}{\varphi} \sin l, \cos l, 0\right), \\
& h_{2}(\lambda)=k \wedge u_{2}(\lambda)=\left(-\frac{1}{\varphi} \sin L, \cos L, 0\right),
\end{aligned}
$$

together with their orthogonal vectors

$$
\begin{aligned}
& h_{1}^{T}(\lambda)=\left(\cos l, \frac{1}{\varphi} \sin l, 0\right), \\
& h_{2}^{T}(\lambda)=\left(\cos L, \frac{1}{\varphi} \sin L, 0\right) .
\end{aligned}
$$

We denote by $\alpha_{i}(\lambda)$ the angle from the vector $k$ to the vector $u_{i}(\lambda)$ for $i=1,2$.

The orientation $M$ is given by the orthogonal matrix obtained as the rotation of angle $\phi$ around the unitary vector $\left(0, v_{2}, 1\right) / \varphi$. So, according with the notation introduced in $(2)$ we have that $M=\mathcal{R}\left(\left(0, v_{2}, 1\right) / \varphi, \phi\right)$.

The orientation $M$ is obtained at the point

$$
P(n, \lambda)=P_{0}+\left(2 n \pi+2 \alpha_{1}(\lambda)\right) \frac{-h_{1}^{T}(\lambda)}{\left\|h_{1}^{T}(\lambda)\right\|}+\left(2 n \pi-2 \alpha_{2}(\lambda)\right) \frac{h_{2}^{T}(\lambda)}{\left\|h_{2}^{T}(\lambda)\right\|},
$$


with this notation we mean that we do two moves for going from the point $P_{0}$ to the point $P(n, \lambda)$, first rotating an angle $2 n \pi+2 \alpha_{1}(\lambda)$ around the vector $-h_{1}(\lambda)$ and after rotating angle $2 n \pi-2 \alpha_{2}(\lambda)$ around the vector $h_{2}(\lambda)$. In fact one can check that

$$
M=\mathcal{R}\left(\frac{h_{2}(\lambda)}{\left\|h_{2}(\lambda)\right\|}, 2 n \pi-2 \alpha_{2}(\lambda)\right) \cdot \mathcal{R}\left(\frac{-h_{1}(\lambda)}{\left\|h_{1}(\lambda)\right\|}, 2 n \pi+2 \alpha_{1}(\lambda)\right) .
$$

We claim that for a sufficiently large integer $n>0$, the closed curve $\{P(n, \lambda): \lambda \in[0,2 \pi]\}$ intersects, at a certain value $\tilde{\lambda}$ of $\lambda$, a circle centered at $P_{0}=(0,0,0)$ (the origin) with radius $2 m \pi$ for some integer $m>0$. At the point $P(n, \tilde{\lambda})$ the orientation is $M$ and rolling the ball along the segment $\left[P_{0}, P(n, \tilde{\lambda})\right]$ with a length $2 m \pi$ we get, at the origin, the same orientation $M$. Therefore, starting at the origin with the identity and doing three moves we come back to the origin with the given orientation $M$. This completes the proof of the theorem. Now we shall prove the claim.

First we shall prove that

$$
\|P(n, \lambda)\|_{\max }-\|P(n, \lambda)\|_{\min } \geq\|P(n, \pi / 2)\|-\|P(n, 0)\|>2 \pi,
$$

for $n$ sufficiently large. The previous maximum and minimum are taking with respect to $\lambda \in[0,2 \pi]$. Indeed from $(7)$ we obtain for $\|P(n, \pi / 2)\|$ and $\|P(n, 0)\|$ the expressions

$$
\begin{aligned}
& \sqrt{\frac{32 A^{2} \cos ^{2}\left(\frac{\phi}{4}\right)+32 n^{2} \pi^{2}\left(1+v_{2}^{2}\right) \sin ^{2}\left(\frac{\phi}{4}\right)}{2+v_{2}^{2}-v_{2}^{2} \cos \left(\frac{\phi}{2}\right)}}, \\
& \sqrt{\frac{32 B^{2} \sin ^{2}\left(\frac{\phi}{4}\right)+32 n \pi B\left(\cos \left(\frac{\phi}{2}\right)-1\right)+\pi^{2}\left(32 n^{2} \sin ^{2}\left(\frac{\phi}{4}\right)+4\left(1+v_{2}^{2}\right)\left(1+\cos \left(\frac{\phi}{2}\right)\right)\right.}{2+v_{2}^{2}+v_{2}^{2} \cos \left(\frac{\phi}{2}\right)}},
\end{aligned}
$$

respectively, where

$$
A=\arccos \left(-\frac{v_{2} \cos \left(\frac{\phi}{4}\right)}{\sqrt{v_{2}^{2}+1}}\right), \quad B=\arcsin \left(\frac{v_{2} \sin \left(\frac{\phi}{4}\right)}{\sqrt{v_{2}^{2}+1}}\right) .
$$

If $n$ is sufficiently large

$$
\begin{aligned}
& \|P(n, \pi / 2)\| \approx \sqrt{\frac{32 n^{2} \pi^{2}\left(1+v_{2}^{2}\right) \sin ^{2}\left(\frac{\phi}{4}\right)}{2+v_{2}^{2}-v_{2}^{2} \cos \left(\frac{\phi}{2}\right)}}, \\
& \|P(n, 0)\| \approx \sqrt{\frac{32 \pi^{2} n^{2} \sin ^{2}\left(\frac{\phi}{4}\right)}{2+v_{2}^{2}+v_{2}^{2} \cos \left(\frac{\phi}{2}\right)}},
\end{aligned}
$$

From

$$
\|P(n, \pi / 2)\|^{2}-\|P(n, 0)\|^{2} \approx \frac{32 n^{2} \pi^{2} v_{2}^{2}\left(2+v_{2}^{2}\right)\left(1+\cos \left(\frac{\phi}{2}\right)\right) \sin ^{2}\left(\frac{\phi}{4}\right)}{\left(2+v_{2}^{2}-v_{2}^{2} \cos \left(\frac{\phi}{2}\right)\right)\left(2+v_{2}^{2}+v_{2}^{2} \cos \left(\frac{\phi}{2}\right)\right)},
$$

it follows that if $n$ is sufficiently large and $\phi \neq \pi$, then

$$
\|P(n, \pi / 2)\|-\|P(n, 0)\|>2 \pi .
$$

So (8) is proved except if $\phi=\pi$. 
For $\phi=\pi$ we have that

$$
\|P(n, \pi / 2)\|^{2}-\|P(n, 0)\|^{2}=\frac{4(2 n+1) \pi\left((2 n-1) \pi v_{2}^{2}+4 \arcsin \left(\frac{v_{2}}{\sqrt{2} \sqrt{v_{2}^{2}+1}}\right)\right)}{v_{2}^{2}+2}
$$

Then for $n$ sufficiently large we again obtain (9). Hence (8) is proved.

Let $n_{0}$ be a positive integer for which (8) holds. Assume that the closed curve $\{P(n, \lambda)$ : $\lambda \in[0,2 \pi]\}$ stays inside the annulus centered at the origin and with boundaries the circles of radius $R$ and $R+2 \pi$. Then

$$
\left\|P\left(n_{0}, \lambda\right)\right\|_{\max }<R+2 \pi, \text { and }\left\|P\left(n_{0}, \lambda\right)\right\|_{\min }>R .
$$

Therefore

$$
\left\|P\left(n_{0}, \lambda\right)\right\|_{\max }-\left\|P\left(n_{0}, \lambda\right)\right\|_{\min }<2 \pi,
$$

in contradiction with (8). In short the closed curve $\{P(n, \lambda): \lambda \in[0,2 \pi]\}$ intersects one of the circles of radius $2 m \pi$ centered at the origin, and the theorem is proved.

We will show that we can go with 3 moves from the state $\left(P_{0},(i, j, k)\right)$ to the state $\left(P_{1},(I, J, K)\right)$ with $k$ and $K$ linearly independent vectors.

Theorem 8. Given $P_{0}, P_{1} \in \mathbb{R}^{2}$ with $P_{0} \neq P_{1}$ and $M \in S O(3)$ with the third column of $M$ different from $\pm k$, we can pass from the initial state $\left(P_{0}, I d\right)$ to the final state $\left(P_{1}, M\right)$ doing 3 moves.

Proof. The proof of this proposition follows essentially the same steps as in the proof of Theorem 7. We will describe the steps which are different.

Let $d$ be the Euclidian distance between the points $P_{0}=(0,0,0)$ and the point $P_{1}$. Let $k$ be a positive integer such that

$$
2 k \pi-2 d>2 \pi
$$

Let $\{P(n, \lambda): \lambda \in[0,2 \pi]\}$ be the closed curve defined in the proof of Theorem 7 . We write $r(n, \lambda)=\|P(n, \lambda)\|$, i.e. the distance of the point $P(n, \lambda)$ to the origin $P_{0}$. Working as in the proof of the second inequality of (8) we obtain

$$
r(n, \pi / 2)-r(n, 0)>2 k \pi,
$$

if $n$ is sufficiently large. Note that in (8) we get $2 \pi$ instead of $2 k \pi$, but in the proof of (8) the difference $r(n, \pi / 2)-r(n, 0)$ can be as large as we want increasing $n$, so this difference can be greater than $2 k \pi$.

Let $r^{*}(n, \lambda)$ be the Euclidean distance from the point $P(n, \lambda)$ to the point $P_{1}$. By the triangle inequality we get that

$$
r(n, \lambda)-d \leq r^{*}(n, \lambda) \leq r(n, \lambda)+d .
$$

Therefore we obtain

$$
\left.r^{*}(n, \lambda)\right|_{\max } \geq r(n, \pi / 2)-d, \quad \text { and }\left.\quad r^{*}(n, \lambda)\right|_{\min } \leq r(n, 0)+d,
$$

where the maximum and minimum are taken over $\lambda \in[0,2 \pi]$. From these last two inequalities, (10) and (11) we have

$$
\left.r^{*}(n, \lambda)\right|_{\max }-\left.r^{*}(n, \lambda)\right|_{\min } \geq r(n, \pi / 2)-r(n, 0)-2 d>2 k \pi-2 d>2 \pi .
$$

So we have obtained an inequality similar to the inequality (8) but this time with respect to the point $P_{1}$ instead of the origin.

Now working as in the last part of the proof of Theorem 7 we get that the closed curve $\{P(n, \lambda): \lambda \in[0,2 \pi]\}$ intersects one of the circles of radius $2 m \pi$ centered at $P_{1}$, and the theorem follows as in the proof of Theorem 7 . 
Clearly the results from Theorem 3 to Proposition 8 show that we can go with at most 3 moves from the state $\left(P_{0},(i, j, k)\right)$ to any other state $\left(P_{1},(I, J, K)\right)$. Hence we have proved that $N \leq 3$.

Remark 9. Since it is easy to see that we cannot reach $\left(P_{0}, R_{3}(a)\right), 0<a<\pi$, from $\left(P_{0}, I d\right)$ with two moves, one concludes that $N=3$.

\section{ACKNOWLEDGMENTS}

The second author is partially supported by a MCYT/FEDER grant number MTM 200803437, by a CICYT grant number 2009SGR 410 and by ICREA Academia. The third author thanks the FCT (Portugal) for the partial support through Program POCTI/FEDER and $\mathrm{PDCT} / \mathrm{MAT} / 56476 / 2004$.

\section{REFERENCES}

[1] L.M.O. Biscolla, Controlabilidade do rolamento de una esfera sobre una superfície de revoluçao, $\mathrm{Ph}$. D., IME, USP, São Paulo, Brasil, 2005.

[2] H. Goldstein, The Euler Angles and Euler Angles in Alternate Conventions, Section 4.4 and Appendix B in Classical Mechanics, 2nd ed. Reading, MA, Addison-Wesley, pp. 143-148 and 606-610, 1980.

[3] J.M. Hammersley, Oxford commemoration ball, Probability, statistics and analysis, Papers dedicated to David G. Kendall on the occasion of his sixty-fifth birthday. Edited by J. F. C. Kingman and G. E. H. Reuter, London Math. Soc. Lecture Note Ser. 79, Cambridge Univ. Press, Cambridge-New York, pp 112-142, 1983

[4] V. JuRdjevic, Geometric Control Theory, Cambridge Univ. Press, 1997.

Universidade Paulista, Rua Dr. Bacelar, 1212, CeP 04026-002 São Paulo, Brasil; and Universidade São Judas Tadeu, Rua Taquari, 546, CEP 03166-000, São Paulo, Brasil

E-mail address: laurabiscolla@terra.com.br

Departament de Matemàtiques, Universitat Autònoma de Barcelona, 08193 Bellaterra, Barcelona, Catalonia, Spain

E-mail address: jllibre@mat.uab.cat

CAMGSD and ISR, Instituto Superior Técnico, UTL, Av. Rovisco Pais, 1049-001, Lisboa, Portugal; and Departamento de Matemática Aplicada, Instituto de Matemática e Estatística, usp, Rua do Matão, 1010-CEP 05508-900, São Paulo, Brasil

E-mail address: wamoliva@math.ist.utl.pt 